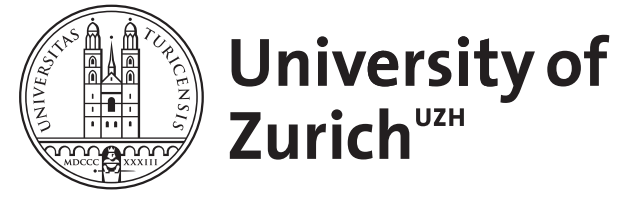

\title{
A remark on the equation of a vibrating plate
}

\author{
Stahel, Andreas
}

\begin{abstract}
SynopsisWe consider the von Karman equations, which describe a vibrating plate either with a clamped boundary or with completely free boundary. In both cases we obtain a unique, classical solution. As the main tool we use a set of integral equations, which we deduce from the well known "variations of constants" formula.
\end{abstract}

DOI: https://doi.org/10.1017/s0308210500018436

Posted at the Zurich Open Repository and Archive, University of Zurich

ZORA URL: https://doi.org/10.5167/uzh-154019

Journal Article

Published Version

Originally published at:

Stahel, Andreas (1987). A remark on the equation of a vibrating plate. Proceedings of the Royal Society of Edinburgh: Section A, 106(3-4):307-314.

DOI: https://doi.org/10.1017/s0308210500018436 


\title{
A remark on the equation of a vibrating plate
}

\author{
Andreas Stahel \\ Mathematisches Institut, Universität Zürich, Rämistrasse 74, CH-8001 Zürich, \\ Switzerland
}

(MS received 25 August 1986. Revised MS received 3 November 1986.)

\section{Synopsis}

We consider the von Karman equations, which describe a vibrating plate either with a clamped boundary or with completely free boundary. In both cases we obtain a unique, classical solution. As the main tool we use a set of integral equations, which we deduce from the well known "variations of constants" formula.

Let $\Omega$ be a bounded smooth domain in $\mathbb{R}^{2}$ with boundary $\Gamma$. We will prove that the following system of equations has classical solutions:

$$
\left.\begin{array}{ll}
\ddot{u}+\Delta^{2} u=[u, \chi], & \text { in } \Omega \times \mathbb{R} \\
D u=0 & \text { on } \Gamma \times \mathbb{R} \\
u(x, 0)=u_{0}, \quad \dot{u}(x, 0)=\dot{u}_{0}, & \text { for } x \text { in } \Omega
\end{array}\right\}
$$

and

$$
\left.\begin{array}{ll}
\Delta^{2} \chi=-[u, u], & \text { in } \quad \Omega \times \mathbb{R} \\
D \chi=0, & \text { on } \Gamma \times \mathbb{R}
\end{array}\right\}
$$

where

$$
[u, v]=\left(\partial_{11} u\right)\left(\partial_{22} v\right)+\left(\partial_{22} u\right)\left(\partial_{11} v\right)-2\left(\partial_{12} u\right)\left(\partial_{12} v\right)
$$

and

$$
D u=\left(\left.u\right|_{\Gamma},\left.\frac{\partial}{\partial v} u\right|_{\Gamma}\right)
$$

denote Dirichlet boundary conditions. This system describes the transversal displacement $u$ and the Airy-stress function $\chi$ of a vibrating plate, whose boundary is clamped in transversal direction but free in horizontal direction (see Landau \& Lifschitz [3]). The best results so far known are due to von Wahl ([6], [7]). He used rather complicated techniques, involving analytic mappings in Banach spaces and nonlinear interpolation theory to prove the existence of strong solutions.

The purpose of this paper is to give a simple method for solving the above problem, which has the advantage of being applicable to other situations where the nonlinearities are not analytic. In addition we can prove the classical solvability of (1) (2), which has not been done before.

First we reformulate the problem by solving (2) and using the solution $\chi$ in (1). 
Let $A=A_{D}$ be the $L^{2}$-realisation of the biharmonic operator $\Delta^{2}$ with Dirichlet boundary conditions. We know that $A$ is a strictly positive, selfadjoint operator with

$$
\operatorname{dom} A_{D}=H_{D}^{4}=\left\{u \in H^{4}(\Omega) \mid D u=0\right\},
$$

and we put

$$
\begin{aligned}
H(u, v, w) & =-\left[u, A_{D}^{-1}[v, w]\right], \\
F(u) & =H(u, u, u), \\
F^{(1)}(u, v) & =H(v, u, u)+2 H(u, u, v) .
\end{aligned}
$$

Now we replace (1) and (2) by the Cauchy problem

$$
\left.\begin{array}{l}
\ddot{u}(t)+A u(t)=F(u(t)), \\
u(t) \in \operatorname{dom} A, \\
u(0)=u_{0}, \quad \dot{u}(0)=\dot{u}_{0} .
\end{array}\right\}
$$

Using well known semigroup theory and a "variations-of-constants" formula we reach (formally) the integral equation

$$
u(t)=\cos \left(t A^{\frac{1}{2}}\right) u_{0}+\sin \left(t A^{\frac{1}{2}}\right) A^{-\frac{1}{2}} \dot{u}_{0}+\int_{0}^{t} \sin \left((t-\tau) A^{\frac{1}{2}}\right) A^{-\frac{1}{2}} F(u(\tau)) d \tau .
$$

Our approach consists in replacing (4) by another system of equations; the main advantage of this is that we have $A^{-1}$ under the integral in place of $A^{-\frac{1}{2}}$. This simple trick allows us to solve the integral equation in the space $\operatorname{dom} A^{\alpha+\frac{1}{2}}$ in place of $\operatorname{dom} A^{\alpha}$, so we have a more regular solution.

To show this, we argue formally as follows. By differentiating (4) and using

$$
\frac{d}{d t} F(u(t))=F^{(1)}(u(t), \dot{u}(t))
$$

we obtain (7). Then we integrate (7) to obtain (6). Thus we arrive at the system

$$
\begin{gathered}
\left.u(t)=\cos \left(t A^{\frac{1}{2}}\right) u_{0}+\sin t A^{\frac{1}{2}}\right) A^{-\frac{1}{2}} \dot{u}_{0}-\cos \left(t A^{\frac{1}{2}}\right) A^{-1} F\left(u_{0}\right)+A^{-1} F\left(u_{0}\right) \\
+\int_{0}^{t}\left(i d-\cos \left((t-\tau) A^{\frac{1}{2}}\right)\right) A^{-1} F^{(1)}(u(\tau), \dot{u}(\tau)) d \tau \\
\dot{u}(t)=-\sin \left(t A^{\frac{1}{2}}\right) A^{\frac{1}{2}} u_{0}+\cos \left(t A^{\frac{1}{2}}\right) \dot{u}_{0}+\sin \left(t A^{\frac{1}{2}}\right) A^{-\frac{1}{2}} F\left(u_{0}\right) \\
\quad+\int_{0}^{t} \sin \left((t-\tau) A^{\frac{1}{2}}\right) A^{-\frac{1}{2}} F^{(1)}(u(\tau), \dot{u}(\tau)) d \tau .
\end{gathered}
$$

The same set of equations was used in [1] and [6]. We thank von Wahl for drawing these papers to our attention. One of the differences in our approach to that of von Wahl's paper is that we do not solve a "weak" problem first and regularise afterwards, but solve (6), (7) in the "best possible" function spaces directly.

Let us consider functions $u$, satisfying

$$
u \in C^{0}\left(J, H_{D}^{4}\right) \cap C^{1}\left(J, H_{D}^{2}\right) \cap C^{2}\left(J, H^{0}\right)
$$


for some nontrivial interval $J$ containing 0 . So we have $F(u().) \in C^{1}\left(J, H^{0}\right)$ and (5) (use Lemma 1 below). Now it is easily verified that the above formal operations are correct and the equations (3) and (6), (7) are indeed equivalent.

Now we shall show that the system (6), (7) possesses a unique solution $(u, v)$ in appropriate function spaces and that $v=\dot{u}$, such that we have a solution to (3). For this we put

$$
H_{D}^{s}=\left\{\begin{array}{lll}
H^{s}(\Omega), & \text { if } & 0<s<\frac{1}{2} \\
H^{s}(\Omega) \cap\left\{u|u|_{\Gamma}=0\right\}, & \text { if } & \frac{1}{2}<s<\frac{3}{2} \\
H^{s}(\Omega) \cap H_{D}^{1} \cap\left\{u\left|\frac{\partial}{\partial v} u\right|_{\Gamma}=0\right\}, & \text { if } \frac{3}{2}<s<\frac{9}{2} \\
H^{s}(\Omega) \cap H_{D}^{4} \cap\left\{u\left|\Delta^{2} u\right|_{\Gamma}=0\right\}, & \text { if } \quad \frac{9}{2}<s<\frac{11}{2} .
\end{array}\right.
$$

If $0<\delta<\frac{1}{2}$ we deduce from this definition and the interpolation results of Grisvard [2]

$$
\begin{aligned}
& \operatorname{dom} A^{\frac{5}{4}+\delta / 4}=H_{D}^{5+\delta} \\
& \operatorname{dom} A^{3+\delta / 4}=H_{D}^{3+\delta} \\
& \operatorname{dom} A^{1+\delta / 4}=H_{D}^{1+\delta},
\end{aligned}
$$

using the well known fact that $\operatorname{dom} A^{\alpha}$ coincide with appropriate interpolation spaces (e.g. $[5,1.18 .10]$ or [4]). Now we easily deduce the following theorem.

THEOREM. Suppose that

$$
u_{0} \in H_{D}^{5+\delta} \text { and } \dot{u}_{0} \in H_{D}^{3+\delta}
$$

for some $\delta \in\left[0, \frac{1}{2}\right)$. Then there exists a maximal interval $J=\left(T^{-}, T^{+}\right)$, containing 0 , and a unique solution $u$ of (3) satisfying

$$
u \in C^{0}\left(J, H_{D}^{5+\delta}\right) \cap C^{1}\left(J, H_{D}^{3+\delta}\right) \cap C^{2}\left(J, H_{D}^{1+\delta}\right)
$$

and by Sobolev embedding theorems this solution is in fact classical. If for some $\varepsilon>0$ and some $k(.) \in C^{0}\left(\mathbb{R}, \mathbb{R}_{+}\right)$

$$
\|u(t)\|_{H^{2+\varepsilon}} \leqq k(t) \text { for all } t \in\left[0, T^{+}\right),
$$

then $T^{+}=\infty$. As long as (8) and $\delta<\frac{1}{2}$ are satisfied, $T^{+}$does not depend on $\delta$. Similar results are true for $T^{-}$.

Proof. From Lemma 1 (i) and Lemma 2 we obtain

$$
F^{(1)} \in C^{1-}\left(H_{D}^{5+\delta} \times H_{D}^{3+\delta}, H_{D}^{1+\delta}\right)
$$

(i.e. $F^{(1)}$ is locally Lipschitz continuous) and that $F^{(1)}$ is bounded on bounded sets. By standard iteration and continuation arguments we obtain a solution $(u, v)$ of $(6),(7)$ satisfying

$$
(u, v) \in C^{0}\left(J, H_{D}^{5+\delta} \times H_{D}^{3+\delta}\right)
$$

and

$$
\lim _{t \rightarrow T^{+}}\left(\|u(t)\|_{H_{D}^{5+\delta}}+\|v(t)\|_{H_{D}^{3+\delta}}\right)=\infty
$$


provided $T^{+}<\infty$. We consider only the right endpoint $T^{+}$of $J$, since similar arguments apply to $T^{-}$. Now we differentiate (6) and obtain $\dot{u}(t)=v(t)$ with $u \in C^{1}\left(J, H_{D}^{3+\delta}\right)$. This gives $F(u().) \in C^{1}\left(J, H_{D}^{1+\delta}\right)$ and (5). Thus $u$ is a solution to (3). Suppose (10) and $T^{+}<\infty$, then we apply part (ii) of Lemma 1 and Gronwall's inequality to the integral equation (7), considered in $C^{0}\left(J, H^{2}\right)$. This gives an a priori bound for $\|\dot{u}\|_{H^{2}}$, i.e.

$$
\sup _{t \in\left[0, T^{+}\right)}\|\dot{u}(t)\|_{H^{2}<\infty}
$$

If we look at the expressions under the integral in (6), we obtain an a priori bound for $\|u\|_{H^{4}}$. Now we find a bound for $\|\dot{u}\|_{H^{3+\delta}}$ by Lemma 1 (i) and Gronwall's inequality applied to (7). Thus, using (6) again, $\|u\|_{H^{5+\delta}}$ is bounded and we have a contradiction to (11).

If $\delta_{1} \leqq \delta_{2}<\frac{1}{2}$ and $T_{1}^{+}$(respectively $T_{2}^{+}$) is the corresponding time of existence, it is obvious that $T_{2}^{+} \leqq T_{1}^{+}$. If $T_{2}^{+}<T_{1}^{+}$, then

$$
\sup _{t \in\left[0, T_{2}^{+}\right]}\|u(t)\|_{H^{4}}<\infty
$$

and we obtain $T_{2}^{+}=\infty$ (by means of (10)). Consequently $T_{2}^{+}=T_{1}^{+}$and the Theorem is proved.

Remarks. (A) Von Wahl proved global existence under one of the assumptions

$$
\|[u(t), u(t)]\|_{L^{p}}<k(t), \quad \text { for all } t \in\left[0, T^{+}\right),
$$

for some $p>1$, or

$$
\|K(t)\|_{L^{p}}<k(t), \text { for all } t \in\left[0, T^{+}\right),
$$

for some $p>1$, where $K$ is the Gaussian curvature of the bent plate (see [6, formula V.12/13]). In [6, p. 71] the author proves that $\left(10^{\prime}\right)$ and $\left(10^{\prime \prime}\right)$ are equivalent if used to prove global existence. We show that $(10)$ and $\left(10^{\prime}\right)$ are equivalent in this context. So it does not matter which of the conditions (10), $\left(10^{\prime}\right)$ or $\left(10^{\prime \prime}\right)$ we use. They are different formulations of the same assumption and all of them imply global existence of the solution $u$.

The proof of part (ii) of Lemma 1 shows that (10) implies $\left(10^{\prime}\right)$ (look at the lower left corner of the first diagram of that proof). Let us assume $\left(10^{\prime}\right)$. If we consider in $F(u)=H(u, u, u)$ only the first argument of $H$ and fix the other two, then we obtain, by the proof of Lemma $1(i)$ and $\left(10^{\prime}\right)$, the estimate

$$
\|F(u)\|_{H^{1}} \leqq c\|[u, u]\|_{L^{p}}\|u\|_{H^{3}} \leqq c k(t)\|u\|_{H^{3}} .
$$

Now we take (4) as an integral equation for $u \in C^{0}\left(J, H^{3}\right)$ and apply Gronwall's inequality. Thus we obtain an a priori estimate for $\|u\|_{H^{3}}$. We see that (10) is satisfied and so is in fact equivalent to $\left(10^{\prime}\right)$.

(B) It is apparently not known whether the above result is true in the limiting case $\delta=\frac{1}{2}$. The above proof does not work, since $H^{\frac{3}{2}}$ is not a subspace of $\operatorname{dom} A_{D}^{\frac{3}{8}}$ (c.f. [2]) and the nonlinearity does not satisfy further boundary conditions (except the one given in Lemma 2). 
(C) The basic idea of the above proof applies to more general situations. Let $A$ be a strictly positive, selfadjoint operator. For a fixed number $\alpha \geqq 0$ we have to find an

$$
F^{(1)} \in C^{1-}\left(\operatorname{dom} A^{1+\alpha} \times \operatorname{dom} A^{\frac{1}{2}+\alpha}, \operatorname{dom} A^{\alpha}\right),
$$

(where $\operatorname{dom} A^{\alpha}$ is endowed with the graph norm), which is bounded on bounded sets such that

$$
u \in C^{0}\left(J, \operatorname{dom} A^{1+\alpha}\right) \cap C^{1}\left(J, \operatorname{dom} A^{\frac{1}{2}+\alpha}\right)
$$

implies

$$
F(u(.)) \in C^{1}\left(J, \operatorname{dom} A^{\alpha}\right) \quad \text { and } \quad \frac{d}{d t} F(u(t))=F^{(1)}(u(t), \dot{u}(t)) .
$$

If this condition is satisfied, by solving (6) (7) we obtain a solution $u$ to (4) with

$$
u \in C^{0}\left(J, \operatorname{dom} A^{1+\alpha}\right) \cap C^{1}\left(J, \operatorname{dom} A^{\frac{1}{2}+\alpha}\right) \cap C^{2}\left(J, \operatorname{dom} A^{\alpha}\right) .
$$

Thus $u$ is also a solution to (3).

This remark corresponds to von Wahl's Theorem II.2 in [6], but the conditions on the nonlinearity are different. It is not obvious which approach is more general (with respect to the nonlinearities). For simplicity we have not included the case of time dependent $A(t)$, as is done in [6].

(D) If we consider the equation for a vibrating plate with completely free boundary we must replace the Dirichlet boundary conditions in (1) (but not in (2)) by

$$
\begin{aligned}
B u & =\left(B_{0} u, B_{1} u\right)=(0,0), \\
B_{0} u & =\left(\frac{\partial}{\partial v}\right)^{2} u+\sigma\left(\left(\frac{\partial}{\partial \mu}\right)^{2} u+\kappa \frac{\partial}{\partial v} u\right), \\
B_{1} u & =\left(\frac{\partial}{\partial v}\right) \Delta u+(1-\sigma)\left(\frac{\partial}{\partial \mu} \frac{\partial}{\partial v} \frac{\partial}{\partial \mu} u\right),
\end{aligned}
$$

where $\sigma \in(0,1 / 2)$ is Poisson's ratio, a constant that depends on the plate material, $\kappa$ is the curvature and $\mu$ the tangential vector of the boundary $\Gamma$. The deviation of this equation is given in [3] (provided some additional calculations are carried out). Now, in the Theorem, we replace $A$ by $A_{B}, F$ by $\tilde{F}$ and $F^{(1)}$ by $\tilde{F}^{(1)}$, where

$$
\begin{aligned}
A_{B} u & =\Delta^{2} u+u, \\
\tilde{F}(u) & =F(u)+u, \\
\tilde{F}^{(1)}(u, v) & =F^{(1)}(u, v)+v .
\end{aligned}
$$

We know that for $\delta<\frac{1}{2}$ (writing $A$ for $A_{B}$ again),

$$
\operatorname{dom} A^{\frac{1}{2}+\delta / 4}=H^{2+\delta}, \quad \operatorname{dom} A^{1+\delta / 4}=H_{B}^{4+\delta}, \quad \operatorname{dom} A^{\frac{3}{2}+\delta / 4}=H_{B}^{6+\delta} .
$$

Together with part (iii) of Lemma 1 we are in the situation of Remark C with 
$\alpha=\frac{1}{2}+\delta / 4$. Therefore we have a solution $u$ of (4) with

$$
u \in C^{0}\left(J, H_{B}^{6+\delta}\right) \cap C^{1}\left(J, H_{B}^{4+\delta}\right) \cap C^{2}\left(J, H^{2+\delta}\right) .
$$

By Sobolev-imbedding theorems we deduce that $u$ is a classical solution of

$$
\begin{gathered}
\ddot{u}+\Delta^{2} u=F(u), \quad \text { in } \Omega \times J \\
B u=0, \quad \text { on } \Gamma \times J \\
u(0)=u_{0} \in H_{B}^{6+\delta}, \quad \dot{u}(0)=\dot{u}_{0} \in H_{B}^{4+\delta} .
\end{gathered}
$$

It remains to prove the lemmas which have been used above.

Lemma 1. Fix $0 \leqq \delta \leqq \frac{1}{2}$ and $\varepsilon>0$. Then

$$
H \in \mathscr{L}^{3}(X \times Y \times Y, Z) \cap \mathscr{L}^{3}(Y \times Y \times X, Z),
$$

(i.e. $H$ is trilinear and continuous) with

(i) $X=H^{3+\delta}, Y=H^{4}, Z=H^{1+\delta}$,

(ii) $X=H^{2}, Y=H^{2+\varepsilon}, Z=H^{0}$,

(iii) $X=H^{4+\delta}, Y=H^{6}, Z=H^{2+\delta}$.

Proof. Let $\partial^{2}$ be a differential operator of order 2 and denote the multiplication of two functions by $\circ$. Then we have to find appropriate function spaces, over $\Omega$, such that in the following two diagrams all mappings are continuous:

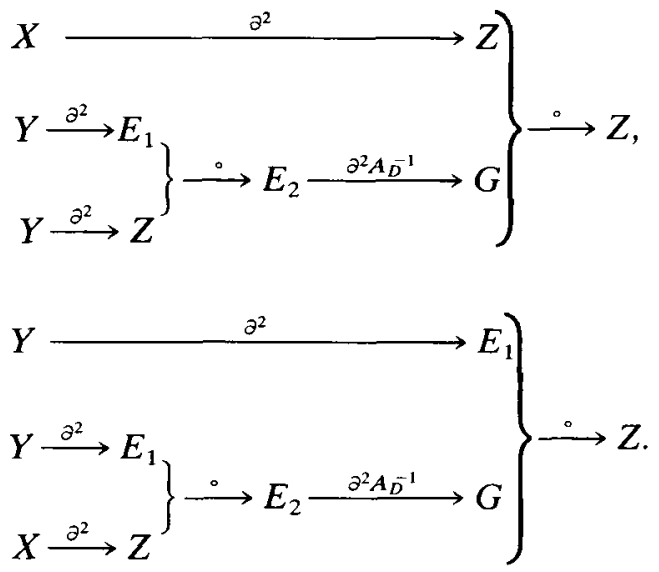

It is not too difficult to see that the following choices are admissible:

(i) $E_{1}=H^{2}, E_{2}=H^{1}, G=H^{3}$,

(ii) $E_{1}=H^{\varepsilon}, E_{2}=L^{p}$ for $1-\varepsilon / 2=1 / p, G=W^{2, p}$,

(iii) $E_{1}=H^{4}, E_{2}=H^{2}, G=H^{4}$.

The following lemma implies that $F(u)$ and $F^{(1)}(u)$ vanish on $\Gamma$ if $u$ and $(\partial / \partial v) u$ are 0 on $\Gamma$. We use this fact in the Theorem to obtain solutions $u \in H^{5+\delta}$, which is better than von Wahl's result [6] [7] (he proved $u \in H^{4+\delta}$ ).

Lemma 2. If $u, v \in H_{D}^{3+\delta}$ then $[u, v] \in H_{D}^{1}$.

Proof. As in the proof of Lemma 1 it is seen that $[u, v] \in H^{1}$. We have to show 
that this expression vanishes on the boundary. Because $\Gamma$ is smooth, we can choose the outer unit normal vector $v$ and a tangential vector $\mu$ defined on a neighbourhood of $\Gamma$ such that

$$
\begin{gathered}
v=\left(v_{1}, v_{2}\right), \quad \mu=\left(\mu_{1}, \mu_{2}\right)=\left(-v_{2}, v_{1}\right), \\
\frac{\partial}{\partial \mu} v=\kappa \mu, \quad \frac{\partial}{\partial \mu} \mu=-\kappa v, \quad \frac{\partial}{\partial v} v=0, \quad \frac{\partial}{\partial v} \mu=0,
\end{gathered}
$$

where $\kappa$ is the curvature of $\Gamma$. If we denote by $D^{2} u$ the matrix of second order partial derivatives of $u$, we obtain by elementary differentiation and matrix calculations

$$
\begin{array}{llrl}
\frac{\partial}{\partial v} \frac{\partial}{\partial v} u & =v D^{2} u v^{T}, & \frac{\partial}{\partial v} \frac{\partial}{\partial \mu} u & =v D^{2} u \mu^{T}, \\
\frac{\partial}{\partial \mu} \frac{\partial}{\partial \mu} u & =\mu D^{2} u \mu^{T}-\kappa \frac{\partial}{\partial v} u, & \frac{\partial}{\partial \mu} \frac{\partial}{\partial v} u & =\mu D^{2} u v^{T}+\kappa \frac{\partial}{\partial \mu} u .
\end{array}
$$

From this and the invariance of the Karman bracket $[u, v]$ under rotations we obtain

$$
\begin{aligned}
{[u, v]=} & \left(\partial_{11} u\right)\left(\partial_{22} v\right)+\left(\partial_{22} u\right)\left(\partial_{11} v\right)-\left(\partial_{12} u\right)\left(\partial_{21} v\right)-\left(\partial_{21} u\right)\left(\partial_{12} v\right) \\
= & \left(v D^{2} u v^{T}\right)\left(\mu D^{2} v \mu^{T}\right)+\left(\mu D^{2} u \mu^{T}\right)\left(v D^{2} v v^{T}\right) \\
& -\left(v D^{2} u \mu^{T}\right)\left(\mu D^{2} v v^{T}\right)-\left(\mu D^{2} u v^{T}\right)\left(v D^{2} v \mu^{T}\right) \\
= & \left(\frac{\partial}{\partial v} \frac{\partial}{\partial v} u\right)\left(\frac{\partial}{\partial \mu} \frac{\partial}{\partial \mu} v\right)+\left(\frac{\partial}{\partial \mu} \frac{\partial}{\partial \mu} u\right)\left(\frac{\partial}{\partial v} \frac{\partial}{\partial v} v\right) \\
& -\left(\frac{\partial}{\partial v} \frac{\partial}{\partial \mu} u\right)\left(\frac{\partial}{\partial \mu} \frac{\partial}{\partial v} v\right)-\left(\frac{\partial}{\partial \mu} \frac{\partial}{\partial v} u\right)\left(\frac{\partial}{\partial v} \frac{\partial}{\partial \mu} v\right) \\
& +\kappa\left(\left(\frac{\partial}{\partial v} \frac{\partial}{\partial v} u\right) \frac{\partial}{\partial v} v+\left(\frac{\partial}{\partial v} u\right)\left(\frac{\partial}{\partial v} \frac{\partial}{\partial v} v\right)\right. \\
& \left.+\left(\frac{\partial}{\partial v} \frac{\partial}{\partial \mu} u\right) \frac{\partial}{\partial v} v+\left(\frac{\partial}{\partial v} u\right)\left(\frac{\partial}{\partial v} \frac{\partial}{\partial \mu} v\right)\right)
\end{aligned}
$$

On $\Gamma$ we have, because of $u \in H_{D}^{3}$,

$$
\frac{\partial}{\partial v} u=\frac{\partial}{\partial \mu} u=\frac{\partial}{\partial \mu} \frac{\partial}{\partial \mu} u=0
$$

and the same is true for $v$. Now it is easy to see that $\left.[u, v]\right|_{\Gamma}=0$ and we have proved the lemma.

\section{References}

1 P. Brenner and W. von Wahl. Global classical solutions of nonlinear wave equations. Math. Z. 176 (1981), 87-121.

2 P. Grisvard. Caractérisation de quelques espaces d'interpolation. Arch. Rational Mech. Anal. 25 (1967), 40-63.

3 L. D. Landau and E. M. Lifschitz. Lehrbuch der theoretischen Physik vii (Berlin: Akademie, 1975).

4 M. Taylor. Pseudodifferential Operators (Princeton: Princeton University Press, 1981). 
5 H. Triebel. Interpolation Theory, Function Spaces, Differential Operators (Amsterdam: North Holland, 1978).

$6 \mathrm{~W}$. von Wahl. On nonlinear evolution equations in a Banach space and on nonlinear vibrations of the clampled plate. Bayreuth. Math. Schr. 7 (1981), 1-93.

7 W. von Wahl. Corrections to my paper: ... (see [6]). Bayreuth. Math. Schr. 20 (1985), 205-209.

(Issued 30 July 1987) 